\title{
Long term performance stability of silicon sensors
}

\author{
R. Mori ${ }^{a, *}$, C. Betancourt ${ }^{\mathrm{a}}$, S. Kühn ${ }^{\mathrm{a}}$, M. Hauser ${ }^{\mathrm{a}}$, I. Messmer ${ }^{\mathrm{a}}$, A. Hasenfratz ${ }^{\mathrm{a}}$, M. Thomas ${ }^{\mathrm{a}}$, K. Lohwasser ${ }^{\mathrm{b}}$, \\ U. Parzefall ${ }^{\mathrm{a}}$, K. Jakobs ${ }^{\mathrm{a}}$
}

${ }^{a}$ Albert-Ludwigs Universität Freiburg, Hermann-Herder Straße 3, 79104 Freiburg im Breisgau, Germany

${ }^{b}$ Deutsches Elektronen-Synchrotron, Platanenallee 6, 15738 Zeuthen, Germany

\begin{abstract}
The HL-LHC investigations on silicon particle sensor performance are carried out with the intention to reproduce the harsh environments foreseen, but usually in individual short measurements. Recently, several groups have observed a decrease in the charge collection of silicon strip sensors after several days, in particular on sensors showing charge multiplication. This phenomenon has been explained with a surface effect, the increase of charge sharing due to the increment of positive charge in the silicon oxide coming from the source used for charge collection measurements. Observing a similar behaviour in other sensors for which we can exclude this surface effect, we propose and investigate alternative explanations, namely trapping related effects (change of polarization), and annealing related effects. Several n-on-p strip sensors, as-processed and irradiated with protons and neutrons up to $5 \times 10^{15} n_{e q} / \mathrm{cm}^{2}$ have been subjected to charge collection efficiency measurements for several days, while parameters like the impedance have been monitored. The probable stressing conditions have been changed in an attempt to recover the collected charge in case of a decrease. The results show that for the investigated sensors the effect of charge sharing induced by a radioactive source is not important, and a main detrimental factor is due to very high voltage, while at lower voltages the performance is stable.
\end{abstract}

Keywords: irradiated silicon sensor, polarization, annealing, charge collection, long term measurements.

\section{Introduction}

The increasing fluence of the planned high energy ${ }^{26}$ physics experiments at e.g. the LHC Upgrade require the ${ }_{28}^{27}$ development of radiation hard detectors maintaining high ${ }_{29}$ Charge Collection Efficiency (CCE) for the foreseen operation time. Communities like the RD50 Collaboration [1] are currently evaluating new strategies. One example is silicon sensors exploiting the avalanche mechanism in a controlled way (Charge Multiplication (CM) [2]).

The charge collection capability of these sensors has been demonstrated for many years, but usually in individual short measurements. Stable performance in standard ${ }_{37}^{36}$ conditions is well known, but in recent observations irradiated strip sensors measured continuously with a ${ }^{90} \mathrm{Sr}$ betasource and kept at more than $1 k V$ bias voltage showed a 39 significant and slow charge collection capability decrease. 40 For example, Altan [3] observed that a p-type sensor irra- 41 diated to the fluence of $10^{15} n_{e q} / \mathrm{cm}^{2}$, biased at $900 \mathrm{~V}$, at 42 $-20^{\circ} \mathrm{C}$ operative temperature, in front of the ${ }^{90} \mathrm{Sr}$ source ${ }^{43}$ used for the charge collection measurements, exhibited a 44 decrease of its CCE from $150 \%$ (CM mode) to less than ${ }^{45}$ $80 \%$ in about two hours. Klanner and Steinbrück [4, ${ }_{46}$ observed for non-irradiated sensors with p-spray and p- 47 stop isolation, biased at $600 \mathrm{~V}$, and irradiated sensors with 48

\footnotetext{
* Corresponding author

Email address: riccardo.mori@physik.uni-freiburg.de
} (R. Mori) p-stop isolation at $1 k \mathrm{~V}$, a significant decrease in CCE. This is accompanied by an increase in the charge sharing between strips, especially in non-irradiated sensors. These studies explain the behaviour with a surface effect. The gamma rays occurring due to Bremsstrahlung of the ${ }^{90} \mathrm{Sr}$ source radiation establish positive charge in the oxide layer, which increases the surface potential and thus causes charge losses from charge sharing. This phenomenon does not explain other cases, where $(i)$ the oxide charge is already saturated from the irradiation before the long term measurements, $(i i)$ the sensor has an efficient p-spray insulation, ( iii) the decrease is too large to come from charge sharing or from effects concerning a thin layer at the surface.

Apart from the source, the high voltage constitutes another potentially detrimental condition imposed on the sensor. A second possible explanation can come from a change in the electric field configuration due to a polarization effect, as commonly observed in large band-gap semiconductors like Diamond [5] or Cadmium-Telluride [6]. The bias voltage can significantly change the defect charge distribution. For example, a change in the effective doping concentration with bias voltage from a bistable defect has been observed 7 . On the other hand, the fact that temperatures around $-20^{\circ} \mathrm{C}$ should be high enough to relax the charge in deep defect levels with a reasonable crosssection in a much shorter time scale, does not support this explanation. For example, the capture cross-section 
of an electron trap $0.3 \mathrm{eV}$ deep, relaxing in more than $10 h_{105}$ at $-20^{\circ} \mathrm{C}$, should be smaller than $10^{-21} \mathrm{~cm}^{2}$. Moreover, 106 Deep Level Transient Spectroscopy (DLTS) and Thermally ${ }_{107}$ Stimulated Current (TSC) measurements, observing de-108 fect relaxations faster than seconds, show that the deepest ${ }_{109}$ defects, even midgap defects, are resolved already at tem-110 peratures lower than $-80^{\circ} \mathrm{C}\left[\mathrm{8}\right.$. Therefore, the time scale ${ }_{111}$ of the observed drops seems too short.

A third explanation would be a high electric field-driven ${ }_{113}$ annealing. In other words, charged defects can move by the ${ }_{114}$ strong electric field. In this case, however, the time scale ${ }_{115}$ seems too short, because movement of defects is discour- ${ }_{110}$ aged at such low temperatures; significant changes with- ${ }_{117}$ out externally applied potential are commonly observed ${ }_{118}$ for example in some weeks at room temperature [9.

The key points to discriminate at least between these ${ }_{120}$ three possible explanations are the following. We need ${ }_{121}$ to determine whether the source or the bias voltage is the ${ }_{122}$ main cause. Then we need to distinguish between bulk $\operatorname{and}_{123}$ surface effects, and eventually find a method to monitor ${ }_{124}$ properties related to loose of efficiency. Finally we should ${ }_{125}$ prove if the initial performance is re-storable modifying ${ }_{126}$ the charge distribution.

\section{Materials}

We tested 10 p-type strip sensors, of about $1 \mathrm{~cm} \times 1 \mathrm{~cm}$ area and all of them with direct biasing (see Table 1). Charge collection measurements have been performed with ${ }^{131}$ the ALiBaVa system [12]. The sensor and read-out board were placed in a freezer where a temperature less $\operatorname{than}^{132}$ $-50^{\circ} \mathrm{C}$ can be reached using a controlled cold nitrogen ${ }^{133}$ vapour flow. ${ }^{90} \mathrm{Sr}$ was used as a source of MIP-like particles ${ }^{134}$ and two scintillators in coincidence provided the trigger signal for the read-out. Humidity and temperature have been monitored with a SHT71 and a PT1000 sensor close to the silicon sensor. The bias voltage has been applied and the current has been monitored by a Keithley 237 or Iseg SHQ222, through an external low pass filter. The sensor impedance was measured using the LCR meter HP 4284A and a custom circuit based on externally controlled relays.

\section{Methods}

Charge collection values were obtained as follows. We performed a clustering procedure summing seed and neighbour channels when having signal to noise ratio higher than 4 and 2, respectively. We then fit the charge distribution with a Landau convolved with a Gaussian function135 and taking the maximum probable value (MPV). The un-136 certainties were computed as the uncorrelated sum of the137 fit uncertainty and the on in the gain of the read-out, a $2 \% 138$ factor on the MPV itself. The cluster size is the number of ${ }_{139}$ channels contributing to the sum, for which a Poissonian 140 error distribution has been assumed.
From capacitance and charge collection measurements versus voltage, we selected the bias voltage to be close to breakdown. For irradiated sensors, it was well below full depletion.

For the initial measurements, we used a non-irradiated detector and we performed a long term measurement maintaining the source in front and removing the bias voltage between charge collection measurements, for several days. In these conditions an eventual decrease can be directly attributed to the surface effect.

We then tried to discriminate between temporary (surface or polarization effects) and permanent (annealing effect) changes. We tested irradiated sensors with both the source in front and the high voltage applied, monitoring the impedance between measurements. The impedance is strictly related to the effective doping concentration and electric field distribution in the bulk. It is well documented how to retrieve defect parameters from the capacitance versus frequency, voltage and temperature characteristics 13] 14. We then performed impedance monitoring every few minutes scanning the allowed frequency range of $20 \mathrm{~Hz}$ to $1 \mathrm{MHz}$. In case of a drop of CCE, we removed the high voltage for an hour, then a day, before performing other measurements to observe eventual recoveries. This would support the temporarily, bulk-related, change and so the polarization explanation.

\section{Experimental results}

In Fig. 1 the charge collection of the as-processed sensor 2 is shown. It was tested at around $23^{\circ} \mathrm{C}$ with the $\mathrm{Sr}$ source always in front and performing measurements from $25 \mathrm{~V}$ to $150 \mathrm{~V}$. The collection saturates at about $75 \mathrm{~V}$

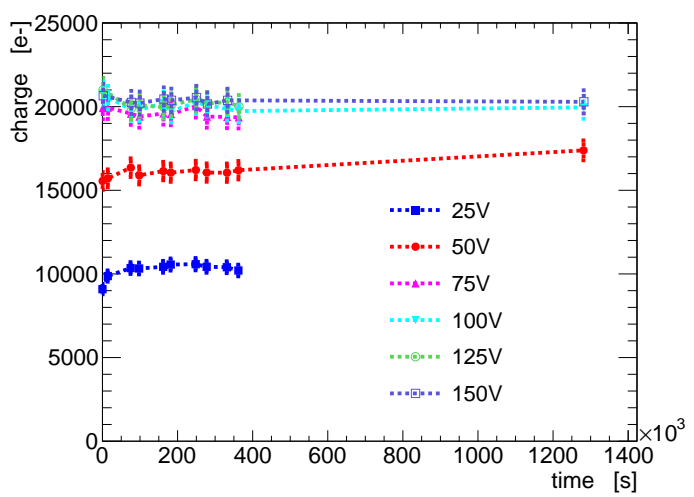

Figure 1: Charge collection versus time at different voltages from the as-processed sensor $2\left(23 \pm 1^{\circ} C\right.$, no voltage between measurements).

to about $22 k e^{-}$, as expected. No decrease is observed in about two weeks.

Several other sensors listed in Table 1 were tested at voltages lower than $1000 \mathrm{~V}$ continuously applied and with the source in front. No significant change of the CCE was observed. 


\begin{tabular}{l|c|c|c|c|c|c|c|} 
Name & Producer & Impl. tech. & Pitch $[\mu \mathrm{m}]$ & Width $[\mu \mathrm{m}]$ & Strip ins. & Thickness $[\mu \mathrm{m}]$ & Fluence $\left[n_{e q} / \mathrm{cm}^{2}\right]$ \\
\hline 1 & HPK & std & 74.5 & 16 & p-stops & 310 & 0 \\
2 & Micron & std & 80 & 25 & p-spray & 300 & 0 \\
3 & Micron & std & 80 & 60 & p-spray & 300 & $1 \times 10^{15}(\mathrm{n})$ \\
4 & CNM & LGAD & 80 & 32 & p-stops & 264 & 0 \\
5 & Micron & std & 80 & 6 & p-spray & 300 & $1 \times 10^{15}(\mathrm{p})$ \\
6 & Micron & std & 80 & 25 & p-spray & 300 & $1 \times 10^{15}(\mathrm{p})$ \\
7 & Micron & inter-strips & 40 & 15 & p-spray & 300 & $1 \times 10^{15}(\mathrm{n})$ \\
8 & Micron & std & 100 & 10 & p-spray & 300 & $5 \times 10^{15}(\mathrm{n})$ \\
9 & Micron & std & 80 & 25 & p-spray & 300 & $5 \times 10^{15}(\mathrm{n})$ \\
10 & Micron & $2 \mathrm{E}$ & 100 & 10 & p-spray & 300 & $1 \times 10^{15}(\mathrm{p})$ \\
\hline
\end{tabular}

Table 1: Sensor specifications. Different implant technologies are indicated: $L G A D$ indicates a multiplication implant under the junction [10, $2 E$ indicates double implantation energy and inter-strips the presence of a floating intermediate strip between the read-out strips [1]. Beside the fluence value, irradiation particles are indicated: $p$ indicates proton, $n$ indicates neutron.

The proton irradiated sample 10 was tested at a bias voltage of $1300 \mathrm{~V}$ for four days, then the voltage was removed for about one week, then applied and tested again; for the full time the source was in front of the sensor, at around $-18^{\circ} \mathrm{C}$. Fig. 2 shows the time evolution of charge collection and of the cluster size of the first four days and after the week of rest. The initial charge of $18 \pm 0.5 \mathrm{ke}^{-}$,
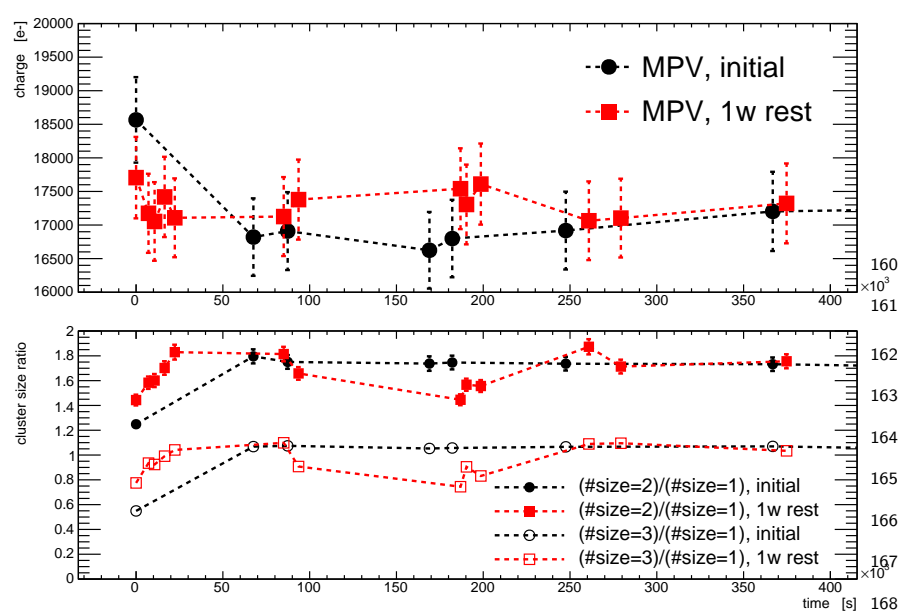

169

Figure 2: (Top) Charce collection versus time and (bottom) clus-170 ter size ratios of the sensor 10 , a sensor proton irradiated to ti1 $_{17}$ $1 \times 10^{15} n_{e q} / \mathrm{cm}^{2}\left(-18 \pm 2^{\circ} \mathrm{C}, 1300 \mathrm{~V}\right.$ continously applied). Cluster ${ }^{171}$ size ratios are defined as the number of events having a cluster size ${ }^{172}$ 2 and 3 over the ones having cluster size 1 (errors are negligible).173 Measurements before (dots) and after (squares) one week of rest. 174

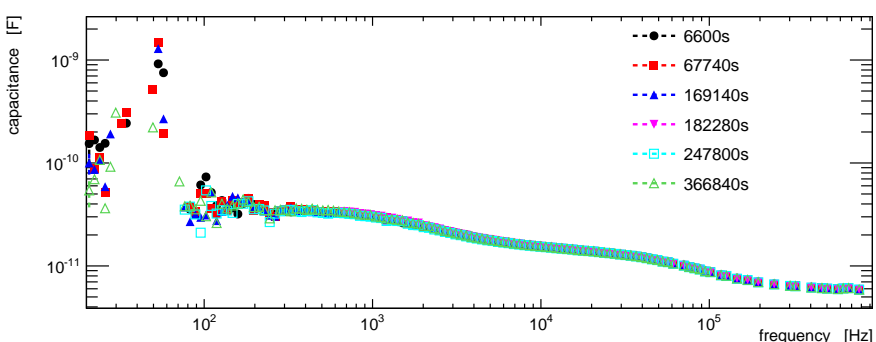

Figure 3: Capacitance versus frequency at different times, of a sensor proton irradiated to $1 \times 10^{15} n_{e q} / \mathrm{cm}^{2}\left(-18 \pm 2^{\circ} \mathrm{C}, 1300 \mathrm{~V}\right.$ continously applied).

geometrical one (around $30 p F$ ) is coming from the strip geometry [15].

Fig. 4 shows the charge collection and cluster size of the neutron irradiated sensor 9. The sensor was tested at $1300 \mathrm{~V}$ with the source continuously in front for more than 10 days, then the voltage was removed for 1 day and applied again, always at around $-40^{\circ} \mathrm{C}$. The sensor collected around $50 \%$ less than an as-processed sample also at the first measurement. This is clearly attributed to expected radiation damage. The collection has a slight initial increase up to about $14 k e^{-}$, then a smooth decrease of more than $2 k e^{-}$. After resting the sensor without bias for a day, the charge remains low.However, a slight increase of charge is seen on the same time scale as for the initial measurement. The clustering behaviour is related to the collection: lower charge is reflected by higher cluster size.

Fig. 5 shows similar results from the neutron irradiated sensor 8. The sensor was tested at around $-40^{\circ} \mathrm{C}$, kept at $1300 \mathrm{~V}$ and with the source in front. During the liquid nitrogen refilling the temperature went up for a maximum of 15 hours, reaching a peak of $-30^{\circ} \mathrm{C}$, compliance was reached and the voltage ramped down to a minimum of $550 \mathrm{~V}$. After about 13 days the voltage was removed for a day, and new measurements were performed for more than 10 days. The sensor was tested again after 8 months in the freezer at around $-10^{\circ} \mathrm{C}$, without voltage and source. The charge collection was initially more than $18 k e^{-}$, with the 

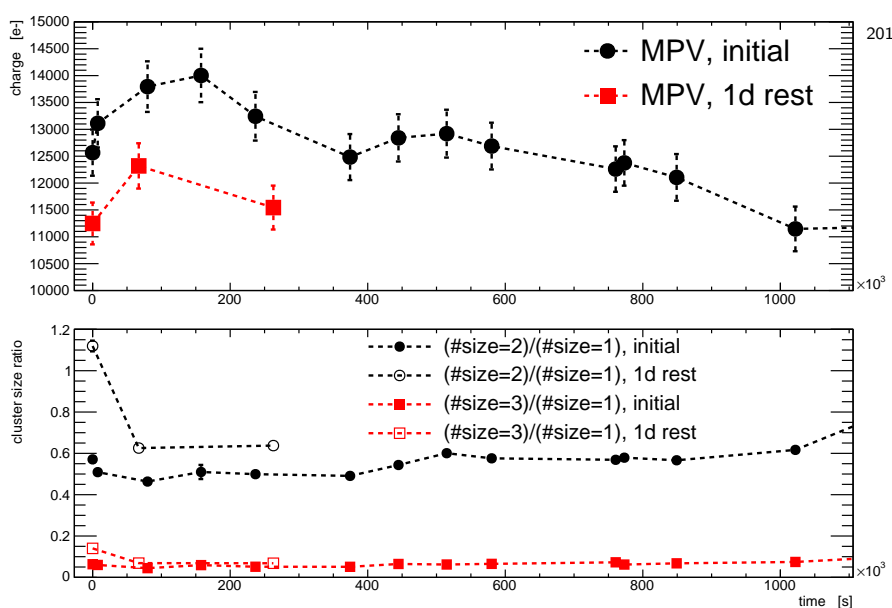

Figure 4: (Top) Charge collection versus time and (bottom) cluster size ratios (2 to 1 and 3 to 1 ) from the sensor 9, a sensor neutron irradiated to $5 \times 10^{15} n_{e q} / \mathrm{cm}^{2}\left(-40 \pm 10^{\circ} \mathrm{C}, 1300 \mathrm{~V}\right.$ continously applied). Measurements before (dots) and after (squares) one day of rest.
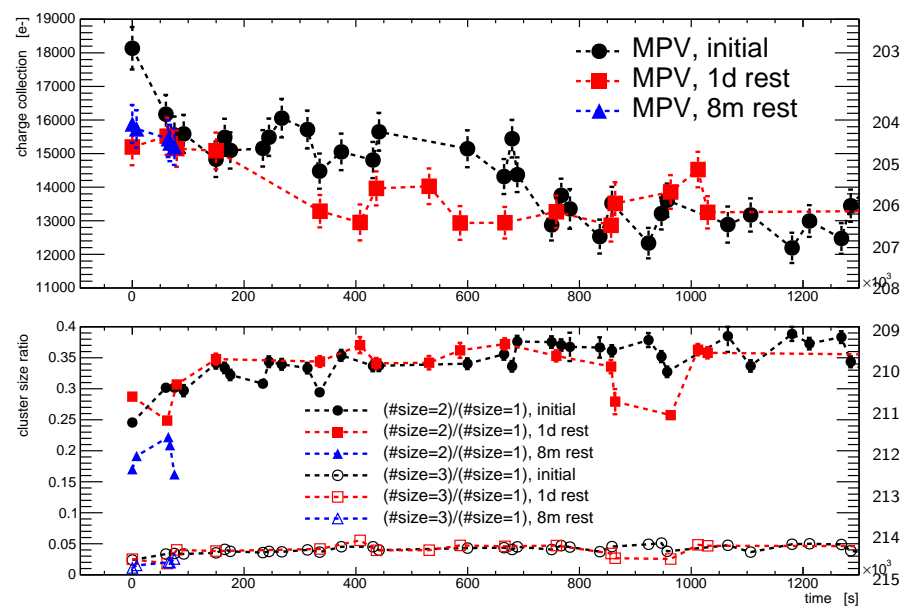

Figure 5: (Top) Charge collection versus time and (bottom) clus- ${ }^{216}$ ter size ratios from the sensor 8 , a sensor neutron irradiated to ${ }^{217}$ $5 \times 10^{15} n_{e q} / \mathrm{cm}^{2}\left(-40 \pm 10^{\circ} \mathrm{C}, 1300 \mathrm{~V}\right.$ continously applied). Mea-218 surements before (dots) and after one day (squares) and 8 months 219 (triangles) of rest. as usually coming in CM regime, that become even broader after the decrease.

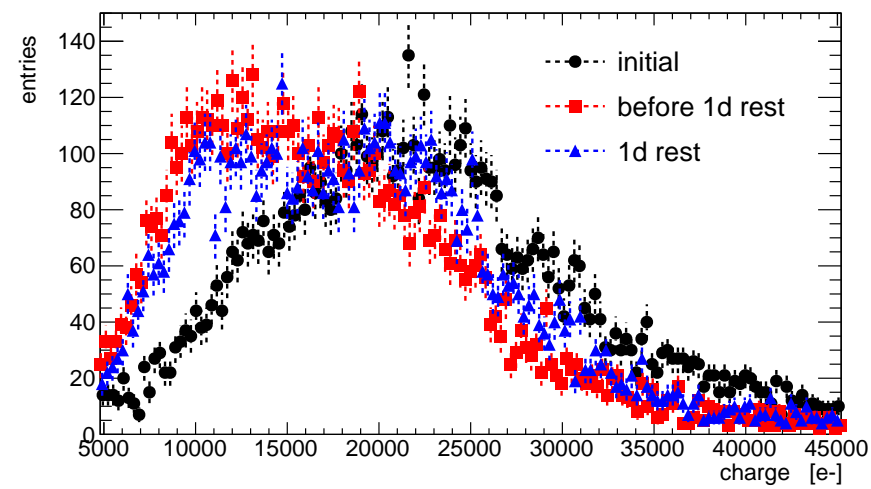

Figure 6: Charge distributions of the sensor 8, a sensor neutron irradiated to $5 \times 10^{15} n_{e q} / \mathrm{cm}^{2}$. The entries are cumulated in a bin of $2 A D C$ width of the read-out electronics, then converted to charge by the gain factor.

\section{Discussion}

Motivation behind samples and set-up. A long term performance decrease has recently been reported in strip sensors showing charge multiplication and kept at bias voltages exceeding $1 k V$. This signal decrease is clearly linked to the high electric field, with the field peaks generally located at the edges of the strip implants. The occurrence of many such edges makes the field also much stronger in strip than in pad sensors.

In order to discriminate between the possible causes of the signal decrease, several properties, primarily the impedance, in parallel with charge collection measurements were studied.

Discrimination between surface and bulk effects. The asprocessed sample was tested at lower voltage with respect to the irradiated ones. As the source-related effects should be evident independently of the bias voltage, this demonstrates that the surface effect is not the cause. Evidently, the p-spray is effective enough in our samples to sufficiently isolate the strips before and after irradiation. Regarding the irradiated detectors, it is worth noting that the neutron irradiation in the core of the Ljubljana reactor also provides a very large gamma ray component which saturates the oxide. In addition, the partial recovery observed for example in the sensors shown in Fig. 2 and Fig. 5 was obtained after some time without bias voltage, but with the source remaining in front of the sensor.

Therefore, also the measurements with irradiated detectors can not be explained by a surface effect. We observed that any signal decrease occurred only in case of bias voltages around $1300 \mathrm{~V}$. Several other sensors (not shown here) exhibit no decreases at lower voltages.

This suggests that key sensor properties change only when the electric field is extremely high. 
Discrimination between polarization and annealing effects.290 Both a polarization and high field-driven annealing would ${ }^{291}$ result in a change in the electric field distribution, visible ${ }_{293}^{292}$ in the capacitance.

The main difference between the two effects is that po-295 larization can be changed in a relatively short time com- ${ }^{296}$ pared to annealing. We observed (Fig. 2 and Fig. 5) $\mathrm{a}_{298}^{297}$ small partial recovery after removing the bias. We can at ${ }_{299}$ this stage not fully exclude that this could originate from 300 a change in the polarization, but the fact that we are not ${ }^{301}$ able to recover the initial charge suggest that most if not $_{303}^{302}$ all of the change is permanent.

Therefore, our observations favour annealing effects. 305 Others have also observed a long term effect of the bias ${ }^{306}$ voltage in the effective doping concentration during an- ${ }_{308}^{307}$ nealing at low temperatures [7].

Temperature influence. Further studies should influence of the temperature on the phenomenon. We ob-312 served that the decrease can happen at relatively differ- ${ }^{313}$ ent temperatures (Fig. 2 vs. Fig. 4 and 5). In any case, ${ }_{315}$ given that the charge distribution in the defects and so the 316 electric field distribution are temperature dependent, we $\mathrm{w}^{317}$ would expect a significant contribution on the amount of ${ }_{319}^{18}$ the charge collection decrease.

\section{Conclusion}

We investigated the causes behind the recently observed ${ }_{325}^{324}$ charge collection decrease on silicon strip sensor, listing ${ }_{326}$ and discriminating between the possible causes.

Surface effects do not explain the observed charge de- ${ }^{328}$ crease: an as-processed sensor in front of the source with- ${ }_{330}$ out bias showed constant performance; in irradiated sen-331 sors a saturated oxide is expected. Partial recovery has ${ }^{332}$ been observed keeping the source in front and removing ${ }_{334}^{333}$ the bias voltage stress. Sensors show an appreciable in- ${ }_{335}$ crease in the cluster size that can be related in this case ${ }^{336}$ also to a weakening of the field at the strip implant corners. ${ }^{337}$

There could be a partial contribution from a polarization effect, as we observed a small partial recovery of the charge removing the voltage. Anyway, the fact that we never recovered the initial charge collection seems to suggest that a major part of the change is permanent. We also observed a correlation between performance decrease and high voltage.

In summary, we can not explain our observations by the surface effect found in similar studies.

Our results suggest that the very high electric field gives rise to a substantial change in the sensor, which could likely be due to a permanent change in the deep defects charge distribution, rather than a reversible polarization effect.

\section{References}

[1] M. Moll, RD50 Status Report 2009/2010 - Radiation hard semiconductor devices for very high luminosity colliders, Tech. Rep.
CERN-LHCC-2012-010. LHCC-SR-004, CERN, Geneva (Jun 2012).

[2] G. Casse, et al., Enhanced efficiency of segmented silicon detectors of different thicknesses after proton irradiations up to $1 * 10^{16} n_{e q} / \mathrm{cm}^{2}$, Nucl. Instr. and Meth. A 624 (2010) 401-404.

[3] L. Altan, Untersuchung zur ladungsmultiplikation an hoch bestrahlten siliziumstreifensensoren, Ph.D. thesis, Institut für Experimentelle Kernphysik (IEKP) (2012).

[4] R. Klanner, Impact of low-dose electron irradiation on the charge collection of $\mathrm{n}+\mathrm{p}$ silicon strip sensors, presented at Technology and Instrumentation in Particle Physics (TIPP14), Amsterdam, Netherlands, 26 June 2014, to appear in PoS.

[5] M. Bruzzi, et al., Improvement of the dosimetric properties of chemical-vapor-deposited diamond films by neutron irradiation, Appl. Phys. Lett. 81 (2) (2002) 298-300.

[6] H. Toyama, et al., Quantitative analysis of polarization phenomena in cdte radiation detectors, Jpn. J. Appl. Phys. 45 (11) (2006) 8842-8847.

[7] M. Mikuž, et al., Bias dependence and bistability of radiation defects in silicon, Nucl. Instr. and Meth. A 466 (2001) 345-353.

[8] I. Pintilie, et al., Radiation-induced point- and cluster-related defects with strong impact on damage properties of silicon detectors, Nucl. Instr. and Meth. A 611 (2009) 52-68.

[9] V. Cindro, et al., Bias-dependent annealing of radiation damage in neutron-irradiated silicon $\mathrm{p}+-\mathrm{n}-\mathrm{n}+$ diodes, Nucl. Instr. and Meth. A 419 (1998) 132-136.

[10] P. Fernández-Martínez, et al., Simulation of new p-type strip detectors with trench to enhance the charge multiplication effect in the n-type electrodes, Nucl. Instr. and Meth. A 658 (2011) 98-102.

[11] C. Betancourt, et al., A charge collection study with dedicated RD50 charge multiplication sensors, Nuclear Instruments and Methods in Physics Research Section A: Accelerators, Spectrometers, Detectors and Associated Equipment 730 (2013) 62 - 65, proceedings of the 9th International Conference on Radiation Effects on Semiconductor Materials Detectors and Devices October 9-12 2012.

[12] R. M. Hernández, A portable readout system for silicon microstrip detectors, Nucl. Instr. and Meth. A 623 (2001) 207-209.

[13] M. Beguwala, et al., Characterization of multiple deep level systems in semiconductor junctions by admittance measurements, Solid-St. Electron. 17 (1974) 203-214.

[14] Z. Li, Modelling of the frequency dependent c-v characteristics of neutron irradiated $\mathrm{p}+-\mathrm{n}$ silicon detectors after the type inversion in the space charge region, IEEE Trans. Nucl. Sci. 41 (4) (1994) 948-956.

[15] E. Barberis, et al., Analysis of capacitance measurements on silicon microstrip detectors, IEEE Trans. Nucl. Sci. 41 (4) (1994) 785-790. 